\title{
Clostridium hastiforme Sepsis in a Patient with Gastrointestinal Malignant Disease
}

\author{
Palash Samanta, Irida Molla, ${ }^{1}$ and Theodore Lenox ${ }^{1,2}$
}

\begin{abstract}
Background: Different Clostridial organisms, commensal in gut flora, can cause blood stream infections, especially in underlying malignant disease. Here, we are presenting first case report of Clostridium hastiforme sepsis in a patient with rectal malignant disease.

Methods: Case report and review of pertinent English-language literature.

Result: A 49-y-old female with known rectal malignant disease was admitted for sepsis with sudden onset sharp rectal pain, fever, leukocytosis, and lactic acidosis. The patient was undergoing palliative chemotherapy. Computed tomography $(\mathrm{CT})$ revealed large rectal carcinoma with central necrosis and distant metastasis. She was started empirically with intravenous vancomycin and piperacillin-tazobactam. Blood culture grew gramvariable rods only in anaerobic bottles, which was subsequently identified as $C$. hastiforme. Antimicrobials were changed to metronidazole. The patient improved clinically and was discharged subsequently.

Conclusions: The report emphasizes the importance of rare Clostridial organisms to cause sepsis in patients with underlying gastrointestinal malignant disease. Patients with this condition are usually gravely ill and the infection can be easily missed if anaerobic culture is not routinely ordered.
\end{abstract}

$\mathbf{S}^{\mathrm{s}}$ EPSIS IN COLORECTAL CANCER is mostly caused by certain bacteria, especially Streptococcus bovis and Clostridial species. Both of them are normal flora in the gastrointestinal tract [1]. Among the Clostridial organisms, several studies reported Clostridium septicum as the causative agent for septic arthritis [2], liver abscess [3], aortitis [4], and sepsis in colorectal cancer [5-8]. Here, we present a case of sepsis caused by Clostridium hastiforme in a patient with anorectal malignant disease to substantiate the importance of other rare Clostridial organisms to cause blood stream infection.

\section{Case}

A 49-y-old Hispanic female, recently diagnosed with metastatic rectal carcinoma, presented to the emergency department with complaints of sudden onset of severe colicky and sharp rectal pain that woke her from sleep in the middle of the night. The pain radiated anteriorly to the lower part of the abdomen and was associated with fever, chills, and palpitations. She also had a few episodes of non-bilious, nonbloody vomiting. She tolerated food and her last bowel movement had been the previous day. She denied any dysuria, diarrhea, constipation, abdominal distension, chest pain, or shortness of breath. She had been admitted to the hospital three months ago and had been diagnosed to have poorly differentiated squamous cell carcinoma of the rectum and anus with metastasis to the liver and adrenal glands. The patient was deemed non-operative and palliative chemotherapy with 5-fluorouracil and cisplatin was begun.

On physical examination the patient was alert and in mild distress for pain. Vital signs were significant for sinus tachycardia and low-grade fever (Tmax $\left.100.2^{\circ} \mathrm{F}\right)$. The abdomen was soft and mildly distended with mild tenderness in the lower abdomen and pelvis; bowel sounds were present. Rectal examination was significant for severe tensemus and a small streak of blood. The examination was incomplete due to severe pain. Laboratory workup showed leukocytosis of 21,040 per microliter with left shift and lactate of $3.3 \mathrm{mmol} /$ L. Computed tomography (CT) scan of the abdomen and pelvis revealed large rectal carcinoma with central necrosis anteriorly with hepatic and adrenal metastasis and probable communication into the vagina, likely a rectovaginal fistula.

\footnotetext{
${ }^{1}$ Department of Internal Medicine, ${ }^{2}$ Division of Infectious Diseases, New York Medical College, Metropolitan Hospital Center, New York, New York.

(C) Palash Samanta et al. 2016; Published by Mary Ann Liebert, Inc. This Open Access article is distributed under the terms of the Creative Commons License (http://creativecommons.org/licenses/by/4.0), which permits unrestricted use, distribution, and reproduction in any medium, provided the original work is properly credited.
} 
Computed tomography also showed the presence of an intrauterine contraceptive device (IUCD) in situ. Blood cultures were sent and the patient was started on intravenous vancomycin and piperacillin-tazobactam and was admitted to intensive care unit for sepsis. Blood cultures were provisionally positive for gramvariable bacilli only in anerobic bottle. Vancomycin was discontinued. Lactate trended down with aggressive intravenous fluid resuscitation. Tachycardia and leukocytosis were resolved. The organism was finally identified as Clostridium hastiforme. Piperacillin-tazobactam was changed to metronidazole. The patient underwent explorative laparotomy followed by palliative diverting loop colostomy. The IUCD was also removed to reduce the risk of further infection. Subsequently the patient improved clinically and was discharged with metronidazole to complete total 14 days of antibiotics.

\section{Discussion}

The genus Clostridium encompasses more than 60 species that may be commensals of the gut microflora or may cause a variety of infections including blood stream infections in human beings. Molecular epidemiologic studies of anaerobic bacteremia have identified $C$. perfringens and $C$. tertium as the two most frequently isolated species; these organisms cause up to $79 \%$ and $5 \%$, respectively, of Clostridial bacteremias [9]. Clostridium septicum is also commonly associated with bacteremia, especially with some gastrointestinal anomaly (e.g., diverticular disease), underlying colorectal malignant disease, diabetes mellitus, and severe atherosclerotic cardiovascular disease. Other Clostridial species, e.g., C. clostridioforme, $C$. hathewayi, $C$. bifermentas, $C$. sordelii, $C$. bolteae, etc. have also been reported rarely to cause bacteremia [9]. To the best of our knowledge, this is the first reported case of $C$. hastiforme causing sepsis in human beings. There are two previous reports of $C$. hastiforme causing eyelid gas gangrene [10] and brain abscess [11]. It has also been reported as colonizer in a fouryear-old male with severe combined immune deficiency [12]. Clostridum hastiforme is a slender, rod-shaped obligate anaerobic organism with rounded ends, $0.3-0.6 \mathrm{mcm}$ in size. The current taxonomy of this organism is Tissierella praeacuta [13]. DNA-DNA hybridization and 16S rRNA gene sequences showed that they seem to be members of same genomic species [14]. They are also indistinguishable based on their biochemical characteristics [13]. Although Clostridia stain grampositive in the early stages of growth, they may appear to be gram-negative or gram-variable later in the growth cycle or in infected tissue specimens leading to the difficulties in choosing the appropriate antibiotics [9]. In the present case also C. hastiforme was provisionally reported as a gram-variable rod. There are few instances in which other bacteria, e.g., Helcococcus kunzii, have been misidentified as C. hastiforme [15]. The clinical importance of recognizing Clostridial bacteremia and starting appropriate treatment immediately cannot be overemphasized. The bacteremia most commonly occurs after hematogenous dissemination usually from ulceration or necrosis in gastrointestinal mucosa. The possible explanation for this association is that the anaerobic glycolysis of the tumor may provide a hypoxic and acidic environment that favors germination of Clostridial spores [16]. These areas of tissue necrosis may explain why gastrointestinal malignant growths have also been reported with Clostridial sepsis. The presence of flagella and the production of various enzymes including fi- brinolysin, hyaluronidase, deoxyribonuclease, and hemolysins also help the Clostridial organisms to invade and spread through the tissue [2]. Thus, patients with this condition usually are gravely ill and infection may metastasize to distant anatomic sites, resulting in spontaneous myonecrosis [9]. Alternative methods to identify these strains, such as polymerase chain reaction (PCR) or other rapid diagnostic tests, are not currently available. The infection can be easily missed if anaerobic culture is not routinely ordered. Anaerobic blood cultures and gram stain interpretation remain the best diagnostic tests at this point. As Clostridial sepsis is an uncommon diagnosis, early identification, administration of appropriate antibiotics, and surgery if possible will improve the outcome.

\section{Author Disclosure Statement}

No competing financial interests exist.

\section{References}

1. Finegold SM, Attebery HR, Sutter VL. Effect of diet on human flora: Comparison of Japanese and American diets. Am J Clin Nutr 1974;27:1456-1469.

2. Dylewski J, Luterman L. Septic arthritis and Clostridium septicum: A clue to colon cancer. Can Med Assoc J 2000; 182:1446-1447.

3. Khan AA, Davenport K. A reminder of the association between Clostridium septicum and colonic adenocarcinoma. Int Semin Surg Oncol 2006;3:12-14.

4. Moseley B, Mwirigi NW, Bowen J. Clostridium septicum aortitis and cecal adenocarcinoma. Case Rep Med 2010;1-3.

5. Mirza NN, McCloud JM, Cheetham MJ. Clostridium septicum sepsis and colorectal cancer-A reminder. W J Surg Oncol 2009;7:72-76.

6. Chew SSB, Lubowski Z. Clostridium septicum and malignancy. ANZ J Surg 2001;71:647-649.

7. Hermsen JL, Schurr MJ, Kudsk KA, et al. PhenotypingClostridium septicum infection: A surgeon's infectious disease. J Surg Res 2008;148:67-76.

8. Mao E, Clements A, Feller E. Clostridium septicum sepsis and colon carcinoma: Report of 4 cases. Case Rep Med 2011;1-3.

9. Bryant AE, Stevens DL. Gas gangrene and other Clostridial infections. In: Longo DL, Kasper DL, Jameson JL, et al. (eds.) Harrison's Principles of Internal Medicine. New York: McGraw Hill, 2012:1204-1210.

10. Lyon DB, Lemke BN. Eyelid gas gangrene. Ophthal Plast Reconstr Surg 1989;5:212-215

11. Cox KA, Al-Rawahi G, Kollman TR. Anaerobic brain abscess following chronic suppurative otitis media in a child from Uganda. Can J Infect Dis Med Microbiol 2009;20:e91-e93.

12. Williamson AP. A special report: Four-year study of a boy with combined immune deficiency maintained in strict reverse isolation from birth. Pediatr Res 1977;11:63-64.

13. Bae JW, Park JR, Chang YH et al. Clostridium hastiforme is a later synonym of Tissierella praeacuta. Int J Syst Evol Microbiol 2004:947-949.

14. Wayne LG, Brenner DJ, Colwell RR, et al. International Committee on Systematic Bacteriology. Report of the ad hoc committee on reconciliation of approaches to bacterial systematics. Int J Syst Bacteriol 1987;37:463-464

15. Woo PCY, Ng KHL, Lau SKP, et al. Usefulness of the MicroSeq 500 16S ribosomal DNA-based bacterial identification system for identification of clinically significant 
bacterial isolates with ambiguous biochemical profiles. J Clin Microbiol 2003;41:1996-2001.

16. Alpern RJ, Dowell VR. Clostridium septicum infections and malignancy. JAMA 1969;209:385-388.

Address correspondence to:

Dr. Palash Samanta

Division of Infectious Diseases Department of Internal Medicine University of Pittsburgh Medical Center Falk Medical Building, Suite $3 A$ 3601 Fifth Avenue Pittsburgh, PA 15213

E-mail: drpalash@gmail.com

\begin{tabular}{|c|} 
Abbreviations Used \\
$\mathrm{CT}=$ computed tomography \\
$\mathrm{IUCD}=$ intrauterine contraceptive device \\
$\mathrm{DNA}=$ deoxyribonucleic acid \\
$\mathrm{rRNA}=$ ribosomal ribonucleic acid \\
$\mathrm{PCR}=$ polymerase chain reaction
\end{tabular}

Cite this article as: Samanta P, Molla I, Lenox T (2016)

Clostridium hastiforme sepsis in a patient with gastrointestinal malignancy. Surgical Infections Case Reports 1:1, 56-58, DOI: 10.1089/crsi.2016. 